\title{
Intervista a Piergiorgio Messa
}

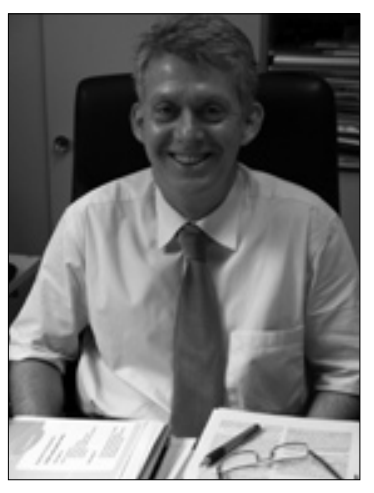

Piergiorgio Messa, MD

Divisione di Nefrologia

e Emodialisi-Croff

pmessa@policlinico.mi.it
Nel centro ove presti attività qual è la percentuale di pazienti monitorati per CKD-MBD stadio 3-5 e quali marker biologici usi a tal fine?

I pazienti seguiti nei nostri ambulatori per CKD 3-5 sono circa 200 . Di questi oltre 1 ' $80 \%$ ha sotto controllo i principali parametri relativi alla CKD-MBD (PTH, Ca, $\mathrm{Pi}, \mathrm{FA})$.

Nel centro ove presti attività pratichi il dosaggio della $25(\mathrm{OH}) \mathrm{D}$ e/o della $1,25(\mathrm{OH})_{2} \mathrm{D} 3$ ?

Se si, in quale tipo di paziente e in quale percentuale? Se no, perché ritieni di non doverla praticare?

Negli ultimi 2 anni, ai parametri sopra elencati abbiamo aggiunto il controllo dei livelli di $25-(\mathrm{OH})-\mathrm{D}$ e solo estemporaneamente quello di 1,25-(OH)2-D.

Applichi la correzione di 25(OH)D in caso di carenza? Perché, con quale protocollo e in quale tipo di paziente?

Ritengo che qualora si evidenzi uno stato di carenza di vitD questo vada corretto in tutti i pazienti, eccettuato quelli con ipercalcemia $(>10,4)$ e/o iperfosforemia $(>5,0)$.

Nel centro ove presti attività in quale percentuale o in quali casi clinici particolari pratichi la biopsia ossea?
Attualmente la biopsia ossea non viene praticata: è comunque in programma la riattivazione di tale indagine.

Nel centro ove presti attività in quale percentuale $e$ in quali stadi di CKD usi indagini strumentali per la ricerca delle calcificazioni vascolari? Quali indagini utilizzi?

Abbiamo già da qualche anno introdotto l'utilizzo della radiografia standard laterale dell'addome per la valutazione dell'indice di calcificazione aortica.

Nel centro ove presti attività quale è la percentuale di pazienti in trattamento sostitutivo che resta nel range di normalità (secondo le linee guida SIN) per Ca, P e PTH?

Con gli attuali schemi di trattamento in uso presso il nostro centro dal 20 al 30\% dei pazienti mantiene in range di normalità tutti e tre i parametri.

Nel centro ove presti attività quale è la percentuale dei vari chelanti usati per ricercare il controllo del Ca-P nei pazienti in trattamento sostitutivo (anche in off-label treatment)?

Oltre 1'80\% dei pazienti necessitano dell'uso di una quota pur minima di chelanti in modo continuativo o discontinuo.

Nel centro ove presti attività quale è la percentuale di pazienti in CKD 3-5 sottoposta a dieta ipoproteica per il controllo della iperfosforemia?

In tutti i pazienti vengono date prescrizioni dietetiche di vario tipo. Una dieta ipoproteica vera e propria viene poi prescritta in circa il 30\% dei casi.

Nel centro ove presti attività quale percentuale di pazienti in trattamento sostitutivo assume vit-D o analoghi, calciomimetico, o entrambi?

Circa il 10\% dei pazienti assume paricalcitolo da solo, $25 \%$ assume una combinazione di cinacalcet + vitD (calcifediolo e/o calcitriolo o paricalcitolo). 
L'iperfosforemia è oramai considerata al pari di altri fattori di rischio noti, come un 'killer' per il paziente uremico. Esistono evidenze sulla capacità di questo elemento nell'indurre meccanismi di variazione fenotipica a livello della cellula muscolare liscia verso cellule capaci di creare matrice ossea e quindi deposizione di fosfato di calcio. $\grave{E}$ il solo fosfato capace di tutto ciò o sono implicati altri mediatori in questo processo? Quale ruolo gioca/no nel processo di ossificazione vascolare? Quali altre novità ci possiamo attendere dalla ricerca in un prossimo futuro?

Numerosi studi sperimentali hanno dimostrato che l'effetto di induzione della metaplasia in senso osteoblastico di vari tipi di cellule mesenchimali (cellule muscolari lisce della parete vascolare, periciti, cellule periavventiziali ecc.) è strettamente legato all'azione di stimolazione diretta da parte del fosforo su un suo specifico trasportatore di membrana (Pit-1), tanto che l'animale KO per tale trasportatore non sviluppa, nelle stesse condizioni sperimentali, il processo di calcificazione vascolare. E comunque da sottolineare che in questi studi le concentrazioni di fosforo utilizzate erano in genere superiori alle $2,0 \mathrm{mmol} / \mathrm{L}$. Tali concentrazioni, sebbene non infrequentemente presenti nel paziente in stadio CKD 5, sono di raro riscontro negli stadi precedenti, quando però le calcificazioni vascolari hanno già iniziato a manifestarsi.

È quindi chiaro che se un nesso patogenetico esiste tra fosforo e calcificazioni vascolari questo andrebbe ricercato non tanto o non solo nella concentrazione di fosfato per sé, ma piuttosto in tutte le alterazioni fisiopatologiche che vengono messe in atto sin dalle fasi più precoci della CKD nel tentativo di mantenere in parità il bilancio del fosfato.

I nuovi attori in tale scenario sono al momento solo in parte definiti (per esempio, FGF23/Klotho), ma molti altri sicuramente si aggiungeranno in un prossimo futuro, come i nuovi fattori fosfaturici di provenienza intestinale.

FGF23. Ł̀ stato detto che nell'uremico avrebbe le carte in regola per diventare un indicatore di esposizione al fosforo... come l'Hb glicata nel diabetico. Cosa ci dobbiamo/possiamo aspettare dall'entrata nell'uso clinico di tale marker?

Se è fuori di dubbio che la definizione di questo ormone fosfaturico ha rappresentato una delle più interessanti acquisizioni dell'ultimo decennio nel campo del metabolismo minerale, molti aspetti rimangono ancora da definire prima di poter assumere che i suoi livelli siano un indicatore fedele dell'esposizione al fosforo. $\mathrm{E}$ da tener presente che i livelli di FGF23 possono essere influenzati da altri fattori, in aggiunta all'apporto fosforico, come la somministrazione di vitamina $\mathrm{D}$, di calcio e dai livelli tessutali di Klotho. Al momento ritengo che la fosfaturia rappresenti un ottimo indicatore dell'apporto dietetico di fosfato.

L'informazione dei livelli di FGF23 potrebbe comunque surrogare una serie di informazioni combinate che potrebbero informarci sullo stato generale di "resistenza" alla capacità di smaltimento di un carico di fosforo.

Solo studi di bilancio totale del fosforo in varie condizioni sperimentali potrebbero però rispondere a tale quesito.

Calciomimetici. Potenti, affidabili, indispensabili: quando come e perché?

L'introduzione dell'uso del calciomimetico ha introdotto due indubitabili vantaggi nella terapia della CKDMBD. Il primo è quello relativo all'aumento delle possibilità di raggiungere contemporaneamente il target dei parametri metabolici (PTH, Ca, $\mathrm{Pi}$ ) rispetto alla terapia tradizionale. Il secondo è quello di aver reso più semplice l'uso della vitamina $\mathrm{D}$ che può essere mantenuta più continuativamente rispetto al passato, grazie al miglior controllo della calcemia e fosforemia che costituivano spesso limiti all'uso protratto della vitamina D.

Rimane ancora irrisolto il problema dei pazienti che manifestano intolleranza gastrica al farmaco, sintomo che talvolta ne condiziona l'uso in modo sostanziale.

Ritengo che un uso più precoce e in associazione con la vitamina $\mathrm{D}$ sia il modo per ottenere la maggiore efficacia nell'uso di questo farmaco. Rimangono però aperti numerosi quesiti di tipo farmaco-economico su quale associazione sia più conveniente e a quali pazienti riservare le associazioni più costose.

Nonostante tutto (linee guida comprese, SIN, K/DOKI, KDIGO) nella maggioranza dei casi i target terapeutici per calcio, fosforo e PTH non vengono centrati e soprattutto mantenuti. Quale commento ti stimola tale insuffciente raggiungimento?

Che il contemporaneo raggiungimento dei target biochimici sia un obiettivo raggiunto in una minoranza dei nostri pazienti, anche con le attuali terapie, è un dato di fatto. Questo apparente poco soddisfacente risultato è legato al fatto che tutte le terapie mediche riescono al meglio a forzare un sistema ampiamente anomeostatico dovuto all'assenza della funzione renale, solo approssimativamente mimata dalla dialisi.

D'altra parte, a parziale consolazione, sappiamo anche che non vi è alcuna dimostrazione prospettica del fatto 
che il mantenimento dei parametri biochimici entro i target desiderati induca un reale vantaggio sugli outcome clinici o quantomeno sui surrogati di outcome (calcificazioni vascolari, ipertrofia miocardica, osteopatia, ecc.).

Non possiamo però negare che, in mancanza di altre informazioni, è certamente più "rassicurante" per il medico e per lo stesso paziente sapere di avere un livello di PTH, di calcemia e di fosforemia più vicini possibile a quelli della popolazione generale. Pertanto, pur in mancanza di certezze, è comunque consigliabile quantomeno tendere a mantenere tali parametri il più possibile nel range della normalità.

\section{Commento conclusivo}

Le nuove acquisizioni nel campo della CKD-MBD hanno ampliato notevolmente la nostra conoscenza in questo campo della fisiopatologia clinica. Oggi sappiamo molto di più rispetto a 20-30 anni fa e abbiamo certamente molti più mezzi terapeutici. Sono però allo stesso tempo aumentati anche i nostri dubbi su quale reale vantaggio clinico derivi dal loro uso. I trials controllati sono ritenuti a (parziale) ragione il mezzo più obiettivo per dissolvere questi dubbi. Non possiamo però non considerare che la quasi totalità dei pochi trials conclusi sino a oggi hanno dato risultati deludenti. Non può non sorgere il dubbio che qualche errore nel disegno di molti di questi studi ha forse concorso a tale risultato negativo. 